\title{
IDENTIFICACIÓN DE NECESIDADES DE APOYO PARA EL APRENDIZAJE Y LA PARTICIPACIÓN DE ESTUDIANTES UNIVERSITARIOS CON DISCAPACIDAD VISUAL: UN ESTUDIO BIOGRÁFICO NARRATIVO
}

\section{Ámbar Núñez ${ }^{1}$, Mauricio López ${ }^{2}$}

\begin{abstract}
RESUMEN
Este artículo explora las experiencias educativas de jóvenes con discapacidad visual, con el objetivo de identificar las necesidades de apoyo para el aprendizaje y la participación experimentada por ellos durante su etapa universitaria. Se utilizó un enfoque biográfico narrativo para explorar la voz de los estudiantes, con la pretensión de que sus perspectivas y valoraciones puedan constituir insumos para la mejora de los procesos de inclusión en la educación superior. Participaron dos estudiantes y dos egresados de dos universidades chilenas y se realizaron dos entrevistas biográficas a cada uno. Las necesidades de apoyo de los estudiantes apuntan a mayor accesibilidad a los espacios para la interacción social, mejores apoyos en movilidad dentro del campus, mejor accesibilidad a los materiales de estudio y contenidos de las clases, y diversificación de las formas de evaluación. Se discuten los resultados a la luz de los conceptos de "participación" y "diseño universal para el aprendizaje", y de las implicancias para las políticas institucionales de inclusión en educación superior.
\end{abstract}

Conceptos clave: Discapacidad visual, necesidades de apoyo, educación superior, accesibilidad, participación.

\section{IDENTIFICATION OF LEARNING SUPPORT NEEDS AND PARTICIPATION OF UNIVERSITY STUDENTS WITH A VISUAL DISABILITY: A NARRATIVE BIOGRAPHICAL STUDY}

\section{ABSTRACT}

This article explores the educational experiences of young people with a visual disability, to identify the needs for learning assistance and the participation they experienced during their university years. A biographical narrative approach was used to explore the students' voices with the intention of having their perspectives and insights contribute to improvements in inclusive education processes in higher education. There was a total of four participants, two current students and two graduates from two Chilean universities and two biographical interviews were conducted with each participant. The students' needs for support include greater accessibility to the areas designated for social interaction, better assistance with mobility within

1 Universidad de Chile, Santiago, Chile. Contacto: ragdoll@uchile.cl

2 Universidad de Chile, Santiago, Chile. Contacto: m.lopez@uchile.cl 
the campus, better accessibility to study materials and class content, and diversification of the assessment instruments. The results are discussed considering the concepts of "participation" and "universal design for learning", and the implications for the institutional inclusion policies in higher education.

Key concepts: Visual impairment, support needs, higher education, accessibility, participation. 
44 IDENTIFICACIÓN DE NECESIDADES DE APOYO PARA EL APRENDIZAJE Y LA PARTICIPACIÓN DE ESTUDIANTES UNIVERSITARIOS CON DISCAPACIDAD VISUAL: UN ESTUDIO BIOGRÁFICO NARRATIVO - Á. Núñez, M. López

\section{Introducción}

La educación superior (ESUP) es considerada el nivel educativo menos inclusivo en términos del acceso, la permanencia y el éxito a largo plazo (Angelino, Kipen, Librandi, Mora y Katz, 2013; PereraRodríguez, Moriña, Cortés y Molina, 2015; Fuller, Bradley \& Healy, 2004; Gitlow, 2001; Hopkins, 2011; Moriña y López-Gavira, 2015; Mullins \& Preyde, 2013; Riddel \& Weedon, 2014), siendo un problema relativamente novedoso en la lucha por los derechos de las personas con discapacidad (Angelino et al., 2013). En Chile, a pesar del aumento que registra la cobertura de ESUP en las últimas décadas —de un 7,5\% en 1980 a un 45,8\% en 2013 en la población entre 18 y 24 años (MINEDUC, 2017)—, el acceso a este nivel educativo continúa siendo inequitativo, lo que se refleja, entre otros elementos, en la falta de apoyos para el acceso de grupos históricamente excluidos (OCDE, 2015), así como para su permanencia y egreso exitoso. Es necesario considerar que los actuales sistemas de educación superior no han sido pensados ni diseñados para responder con plena equidad a la diversidad del alumnado; en los contextos universitarios pueden hallarse múltiples barreras a la presencia, participación y logro de estudiantes con discapacidad, o con otras características que resulten en inequidad (Sandoval, Simón, Márquez y Sánchez, 2019). Por lo tanto, para favorecer la equidad, las instituciones de ESUP deben abordar una serie de necesidades propias, mediante el apoyo de especialistas, la asesoría u otros recursos, y contando con un compromiso institucional sostenido en el tiempo que permita el aprendizaje de la institución.

En ese sentido, en los últimos años han surgido iniciativas que, en la forma de programas de acción afirmativa, promueven el acceso a la ESUP de estudiantes de grupos socioeconómicos desfavorecidos, vías de acceso especial para estudiantes de origen indígena, reducción de brechas de género en áreas profesionales con fuerte presencia masculina o femenina, y programas de apoyo al acceso y la permanencia de estudiantes con discapacidad. Entre estos últimos se encuentran el Programa para la Inclusión de Alumnos con Necesidades Especiales (PIANE), de la Pontificia Universidad Católica de Chile; el Programa de Apoyo Académico para Estudiantes con 
Discapacidad (PAAED), de la Universidad de la Frontera; el Programa de Apoyo a Estudiantes con Discapacidad (PAED), de la Universidad de Chile, y la admisión especial para estudiantes en situación de discapacidad, de la Universidad Católica de Temuco, que data del año 2007 (CINDA, 2019), motivado por la desventaja de estos estudiantes para rendir la Prueba de Selección Universitaria (PSU). A propósito de esta prueba, para el proceso de postulación 2020 se implementó la posibilidad de rendirla con "ajustes, adecuaciones o apoyos", que debían ser solicitados por los postulantes al momento de inscribirse $e^{3}$. Además, durante 2019, las facultades de Ciencias Sociales y de Derecho de la Universidad de Chile — con financiamiento del Servicio Nacional de la Discapacidad (SENADIS) — implementaron un programa de acompañamiento a equipos docentes, que contempló capacitaciones en temas de inclusión y discapacidad, observación de prácticas docentes en aula, acompañamiento a tutores y tutoras (que trabajarán directamente con estudiantes con discapacidad) y, finalmente, evaluación y sistematización de la experiencia.

Todos los programas mencionados son iniciativas aisladas y no constituyen un reflejo del escenario más amplio de la ESUP, a pesar del marco legal vigente. Chile ratificó en 2008 la Convención de la Organización de las Naciones Unidas sobre los Derechos de las Personas con Discapacidad, en cuyo artículo 24 se reconoce su derecho a la educación y el deber del Estado de asegurar que ésta tenga un carácter inclusivo en la modalidad terciaria. Asimismo, la Ley 20.422 sobre igualdad de oportunidades e inclusión social de personas con discapacidad (2010) establece que las instituciones de ESUP deben contar con mecanismos que faciliten el acceso de las personas con discapacidad y adaptar para ellos los medios de enseñanza y los materiales de estudio (art. 39).

Actualmente, se sabe que la discapacidad visual comprende tanto la ceguera como la deficiencia visual, abarcando un rango amplio de condiciones y capacidades (OMS, 2013). En general, para determinarla se considera el parámetro de la agudeza visual

3 Información disponible en la página web del Departamento de Evaluación, Medición y Registro Educacional (DEMRE), Universidad de Chile: https://demre.cl/inscripcion/ postulantes-con-discapacidad 
(habilidad para discriminar detalles finos en objetos o símbolos a una distancia determinada) y la medida de campo visual (capacidad para percibir el espacio físico visible cuando el ojo está mirando a un punto fijo) (Caballo y Núñez, 2013). No obstante, la OMS considera fundamentalmente el criterio de agudeza visual, del cual se derivan las categorías de "discapacidad visual moderada", "discapacidad visual severa" y "ceguera". Sin embargo, estas descripciones médicas se consideran insuficientes en el campo educativo, en el que se opta por utilizar clasificaciones funcionales, que abordan el desempeño visual real durante la realización de actividades (Douglas, McCall, McLinden, Pavey, Ware \& Farrell, 2009), tal como en la categorización de "ciego total", "ciego parcial", "baja visión” y "visión límite" (ONCE, 2012).

El desarrollo de las personas con discapacidad visual tiene ciertas particularidades relevantes para comprender sus trayectorias educativas. Los niños ciegos pequeños adolecen de falta de motivación para explorar los objetos de su entorno, pues no los ven, lo que retrasa o dificulta logros evolutivos como el gateo y la marcha, la adquisición de la noción de permanencia de objeto y los conceptos relacionados con el tamaño de objetos muy grandes, muy pequeños o imposibles de aprehender mediante el tacto, así como el desarrollo del pensamiento abstracto (Warren, 2000, 2002). Respecto del desarrollo social, suele observarse ausencia de sonrisa social y reducción de la expresividad facial, lo que puede afectar el desarrollo del vínculo de apego, dificultad en la intersubjetividad secundaria y dificultades en la adquisición del lenguaje, tales como verbalismos, ecolalias y retrasos en el uso correcto de pronombres (Caballo y Núñez, 2013). No obstante, hacia los 14 o 15 años pueden lograr un sistema de representación mental funcionalmente equiparable a los niños videntes (Ibíd.). Asimismo, para el progreso en el aprendizaje, las personas con discapacidad visual necesitan sistemas de apoyo a la representación (CAST, 2018) que posibiliten su acceso al conocimiento visual, o tiflotecnologías, tales como softwares lectores de pantalla y archivos de audio (Pegalajar, 2013). La relevancia de estos sistemas externos de representación para el aprendizaje radica en que, además de ser soportes del conocimiento, crean nuevas posibilidades, competencias y funciones cognitivas (Pozo, 2006). 
De ahí que el concepto de "necesidades de apoyo" resulte clave para entender los procesos de inclusión. Los apoyos son "recursos y estrategias cuyo propósito es promover el desarrollo, la educación, los intereses y el bienestar personal, y que mejoran el funcionamiento individual” (Luckasson et al., 2002, p. 151). Las necesidades de apoyo, en tanto, constituyen un "constructo psicológico que hace referencia al patrón e intensidad de los apoyos necesarios para que una persona participe en actividades relacionadas con un funcionamiento humano estándar" (Thompson et al., 2010, p. 9).

Los estudios que han abordado la situación de la inclusión de estudiantes con discapacidad en la universidad han identificado necesidades de apoyo asociadas a barreras y recursos del contexto. "Barreras para el aprendizaje y la participación" es un término que se adopta en el Índice de inclusión (Booth y Ainscow, 2000) para hacer referencia a las dificultades que experimenta cualquier estudiante. Se considera que las barreras al aprendizaje y la participación surgen de la interacción entre los estudiantes y sus contextos; las personas, las políticas, las instituciones, las culturas y las circunstancias sociales y económicas que afectan a sus vidas (p. 8). Lo anterior es coherente con un cambio de paradigma desde el modelo médico de la discapacidad — que la reducía a su dimensión fisiológica y, por tanto, competencia solo de la medicina con un foco en la rehabilitación (AAIDD, 2011; Parrilla, 2002; Verdugo y Schalock, 2013)— hacia el modelo social, que sitúa las dificultades asociadas a la discapacidad en la interacción entre la persona y su entorno (AAIDD, 2011; Hugues y Paterson, 2008; Parrilla, 2002; Verdugo y Schalock, 2013).

Entre las barreras más habituales figuran el exceso de clases expositivas o con apoyos solamente visuales, la falta de acceso a tecnologías de apoyo al aprendizaje (López-Gavira, et al., 2016; Lorenzo-Lledó, Lorenzo, Lledó \& Pérez-Vásquez, 2020) y el desconocimiento por parte del profesorado de normativas que reconocen derechos a los estudiantes con discapacidad (Moriña y\& López-Gavira, 2015; Perera-Rodríguez et al., 2015). Entre los recursos se señala, por ejemplo, que los profesores conozcan las necesidades de apoyo de los estudiantes con discapacidad (Moriña \& LópezGavira, 2015; Perera-Rodríguez et al., 2015; Sandoval, Morgado y 
48 IDENTIFICACIÓN DE NECESIDADES DE APOYO PARA EL APRENDIZAJE Y LA PARTICIPACIÓN DE ESTUDIANTES UNIVERSITARIOS CON DISCAPACIDAD VISUAL: UN ESTUDIO BIOGRÁFICO NARRATIVO - Á. Núñez, M. López

Doménech, 2020). Específicamente, los estudios sobre necesidades de apoyo de estudiantes con discapacidad visual identifican ajustes razonables para las evaluaciones de las asignaturas (Da Silveira \& Dos Santos, 2016; Sandoval et al., 2020), orientación y movilidad (Lourens \& Swartz, 2016; Southcott \& Opie, 2016), igualdad en la sala de clases y tecnologías de apoyo al aprendizaje (Southcott $\&$ Opie, 2016), importancia de los compañeros para superar obstáculos y la necesidad de combatir el aislamiento social (Lourens \& Swartz, 2016; Moriña \& López-Gavira, 2015), ser reconocido y apoyado por algunas personas importantes en el entorno social (Langorgen \& Magnus, 2018) y que las estrategias de enseñanza incluyan la posibilidad de trabajar en grupo (López-Gavira et al., 2016). Los estudiantes señalan la necesidad de accesibilidad en los entornos universitarios, mejorar el acceso a la información, procesos de transición a la ESUP planificados, una actitud positiva del profesorado, desarrollo de prácticas inclusivas para clases más participativas y activas, uso de las tecnologías y la formación del profesorado en inclusión y discapacidad, y servicios de orientación laboral (Moriña y Carballo, 2020).

Todo lo anterior es coherente con el Diseño Universal para el Aprendizaje (DUA) (CAST, 2018), que puede ser servir como marco de referencia para conceptualizar las diferentes necesidades asociándolas a sus principios y pautas (Díez y Sánchez, 2015; Sánchez-Gómez y López, 2020). Por Diseño Universal (DU) se entiende el diseño de productos, entornos, programas y servicios que puedan utilizar todas las personas, en la mayor medida posible, sin necesidad de adaptación ni diseño especializado (Convención Internacional sobre los Derechos de las Personas con Discapacidad, 2006). La utilización de este enfoque en la planificación de la enseñanza ha demostrado ser clave en el aumento en la participación de personas con discapacidad en la ESUP (Ruiz, Solé, Echeita, Sala y Datsira, 2012; Sánchez, Díez, Verdugo, Iglesias y Calvo, 2011; Yuval, Procter, Korabik \& Palmer, 2004) y en el aumento de la autoeficacia académica de los estudiantes (Yuval et al., 2004), además de generar beneficios en toda la comunidad universitaria (Darr \& Jones, 2008; Díez y Sánchez, 2015; Rose, Harbour, Johnston, Daley \& Abarbanell, 2006). Asimismo, en la Convención de los derechos 
de personas con discapacidad se propuso el principio de "ajustes razonables", entendido como las modificaciones y adaptaciones necesarias y adecuadas que no impongan una carga desproporcionada o indebida cuando se requieran en un caso particular, para garantizar a las personas con discapacidad el goce o ejercicio, en igualdad de condiciones con las demás, de todos los derechos humanos y libertades fundamentales (p. 5).

Por otra parte, la investigación sobre inclusión en la ESUP requiere dar un lugar central a la voz de sus protagonistas. De acuerdo con Hutcheon y Wolbring (2012), los estudiantes con discapacidad han estado subrepresentados en las facultades universitarias, lo que es consecuencia del discurso y del conocimiento dominantes que invisibilizan la discapacidad. En este sentido, muchos autores coinciden en que los estudiantes excluidos son "voces perdidas", que deben ser escuchadas y que constituyen una fuente valiosa de información en tanto perspectiva en primera persona (Moriña \& López-Gavira, 2015) para el desarrollo de políticas y programas que incidan positivamente en su calidad de vida (López-Gavira et al., 2016). A partir de estos antecedentes, el presente estudio tiene como objetivo identificar las necesidades de apoyo de estudiantes con discapacidad visual en la universidad desde una perspectiva en primera persona, a partir del relato de sus trayectorias educativas y sus experiencias actuales como estudiantes universitarios.

\section{Método}

Este estudio formó parte de una iniciativa del equipo de investigación sobre Inclusión Educativa del Departamento de Psicología de la Universidad de Chile, en el que participan los autores. Se desarrolló en el marco de la implementación de un programa de acción afirmativa para el ingreso de estudiantes con discapacidad visual en la carrera, amparado en la Política de Equidad e Inclusión Estudiantil de la Universidad, aprobada por el Senado Universitario (2014). No se contó con financiamiento directo, pero se dedicaron horas de la carga académica de uno de los autores para la realización de este estudio. 
50 IDENTIFICACIÓN DE NECESIDADES DE APOYO PARA EL APRENDIZAJE Y LA PARTICIPACIÓN DE ESTUDIANTES UNIVERSITARIOS CON DISCAPACIDAD VISUAL: UN ESTUDIO BIOGRÁFICO NARRATIVO - Á. Núñez, M. López

\section{Diseño y enfoque}

Esta investigación adoptó un enfoque cualitativo, con un alcance exploratorio y descriptivo, y un diseño biográfico narrativo. El método biográfico narrativo permite indagar en la forma en que las personas experimentan el mundo (Connelly \& Clandinin, 2004) y acceder a una selección de recuerdos y sucesos y su interpretación (Buontempo, 2000).

\section{Participantes}

Se optó por utilizar muestro teórico, en el cual lo importante es "el potencial de cada caso para ayudar al investigador en el desarrollo de comprensiones teóricas sobre el área estudiada de la vida social" (Taylor y Bogdan, 2002, p. 108). Para constituir el grupo de participantes usamos la técnica de "bola de nieve": en primer lugar, contactamos a un estudiante referido por conocidos y, a partir de éste, cada participante nos refirió a un conocido suyo que cumplía las condiciones de ser o haber sido estudiante universitario con discapacidad visual. Participaron dos estudiantes y dos egresados de dos universidades chilenas de la ciudad de Santiago. Dos cursaban la carrera de Derecho, uno era egresado de la carrera de Pedagogía en Lenguaje y Comunicación, y uno egresado de la carrera de Psicología. Una de las universidades contaba con un programa de apoyo al acceso y permanencia de estudiantes con discapacidad y en la otra universidad no existía un programa similar, pero se encontraba en desarrollo uno en cuya formación colaboraban dos participantes de este estudio. Gaspar nació con ceguera, Sabina quedó con baja visión (30\% de visión) a los meses de vida, Alfonso quedó ciego a los tres años y, finalmente, Daniel quedó ciego a los 15 años, pudiendo realizar la mayor parte de su escolaridad como vidente. Tanto Gaspar como Sabina y Alfonso contaron con el apoyo de una escuela especial durante su escolaridad: asistieron a ella de manera exclusiva en algunos periodos (por ejemplo, durante la enseñanza básica) y, en otros, acudían en busca de apoyo mientras estudiaban en escuelas regulares. En la tabla 1 se describen brevemente los participantes, utilizando nombres ficticios para ello. 
Tabla 1

Participantes del estudio

\begin{tabular}{|c|c|c|c|c|}
\hline Nombre & Edad & Género & Carrera & Situación \\
\hline Alfonso & 27 & Hombre & Derecho & Estudiante \\
\hline Daniel & 28 & Hombre & Derecho & Estudiante \\
\hline Gaspar & 31 & Mujer & Pedagogía en Lenguaje y Comunicación & Egresado \\
\hline Sabina & 28 & Mujer & Psicología & Egresada \\
\hline
\end{tabular}

Técnicas y procedimiento para la producción de datos

Para la producción de datos se planificaron dos encuentros con cada participante. En la primera sesión de entrevista biográficonarrativa (Bolívar, Domingo y Fernández, 2001) se indagó en la historia escolar de los participantes, experiencias influyentes para su trayectoria educativa, elección de carrera, alternativas de acceso e ingreso a la universidad. Tras un primer análisis, en la segunda entrevista se invitó a los participantes a diseñar una universidad ideal (Moriña, López-Gavira y Molina, 2017) en la que sus necesidades de apoyo estuviesen satisfechas. Por ello, en algunos casos, las formas verbales usadas por los participantes están en presente y afirmativo, aun cuando se trate de vivencias y descripciones imaginadas. Las entrevistas fueron realizadas por uno de los autores (persona sin discapacidad) en dependencias de la Universidad, y cada una de ellas tuvo una duración de entre noventa minutos y dos horas.

Procedimiento de análisis de información

Se realizó un análisis de contenido de acuerdo con la teoría fundamentada (Strauss y Corbin, 2002), para lo cual se utilizó el programa ATLAS.TI. En la denominación de los códigos y categorías se conservaron las expresiones utilizadas por los participantes. El material se fue organizando de manera iterativa a lo largo de todo el proceso, siguiendo un modelo interactivo de reducción de datos (Miles y Huberman, 1994), de manera que, luego de realizar la primera codificación, se buscó agrupar aquellos códigos que hicieran referencia a un mismo fenómeno o que tuviesen elementos en común. El siguiente paso fue agruparlos en conjuntos de "códigos conceptuales" para, posteriormente, realizar la codificación axial, que permitió crear redes de relaciones entre los códigos. 
Finalmente, con relación a las consideraciones éticas, a los participantes se les garantizó la confidencialidad y el anonimato, por lo que sus nombres fueron cambiados y dieron su consentimiento informado por escrito. Este estudio no contó con la evaluación del Comité de Ética de la Facultad.

\section{Resultados}

En primer lugar se presentan las experiencias relevantes durante la trayectoria educativa. En segundo término, las necesidades de apoyo referidas a orientación, movilidad y acceso al currículo. Finalmente se explora la idea de accesibilidad universal como política institucional en educación superior.

\section{Experiencias relevantes durante la trayectoria educativa}

Los participantes manifiestan un deseo de independencia que los acompaña desde la enseñanza básica, ligado a la necesidad de participar de los espacios de la vida escolar y de la vida social como niños y adolescentes. En ese horizonte, la posibilidad de adquirir herramientas para el desenvolvimiento cotidiano autónomo se instala en primer plano. Por lo tanto, valoran las experiencias de escolarización que complementan escuela regular y escuela especial, en las que pueden acceder a material de estudio adaptado y adquirir competencias, tales como el uso del bastón para desplazarse y la escritura braille, sin perder por ello la oportunidad de compartir con compañeros sin discapacidad:

... iba una vez a la semana al [colegio] Hellen Keller y ahí me hacian como reforzamiento, aprendi a escribir braille, a usar un poco el bastón. Y me ayudaban, principalmente a hacer cosas, a leer un poco más rápido con letra más grande, eso. (Sabina, primera entrevista)

El no tener acceso a este tipo de apoyo es resentido por los participantes, sobre todo en la enseñanza media, en la que algunos sienten que su entorno los protege más que a sus compañeros sin discapacidad, y carecer de herramientas es un obstáculo para cambiar dicha situación. La enseñanza media aparece como una etapa crítica 
para el avance y el logro de esa anhelada autonomía cotidiana, puesto que es el momento en que normativamente los adolescentes buscan cierta independencia en una serie de actividades, y porque, además, esta transición supone cambio de colegio y con ello nuevas necesidades de desplazamiento, nuevas actividades y nuevos grupos sociales de referencia. Como Sabina detalla:

... en la media aprendí a andar en micro, es que ahí empecé a ir a la Corporación para ciegos, ahí me juntaba con mis amigos, habia talleres de computación, de yoga, todo en el mismo lugar, es increible. La primera vez que me dicen "salgamos a tomarnos un helado uno de estos días”, yo dije sí, obvio, pero por dentro iuy qué miedo! Estaba muy preocupada de cómo lo íbamos a hacer, cómo íbamos a cruzar la calle ... y ahí empecé a abrirme un poco más. (Sabina, primera entrevista)

Parte de la experiencia en la vida universitaria se vincula al desarrollo de intereses profesionales u ocupacionales. En la biografía de todos los participantes aparece la dificultad de ver las fórmulas algebraicas en asignaturas como matemática y química, y de asociar las explicaciones verbales con el correlato material en biología, lo que implica una restricción del acceso al conocimiento por barreras en los sistemas de representación utilizados en clase. Daniel, que perdió la vista a los 15 años, manifiesta que su relación con las asignaturas de la escuela cambió radicalmente a partir de ese momento:

... hasta primero medio me gustó mucho la biología, la química y la física. Ya después, cuando perdí la visión, me costó mucho retomar el tema de esas áreas, en especial la química y la biología. Entonces, me fui quedando con los ramos más humanistas; en los ramos matemáticos me costaba mucho... con braille solo se puede aprender la matemática básica, así que no fue mucho lo que pude aprender. (Daniel, primera entrevista)

El caso de los demás entrevistados tiene matices, en el sentido de que su discapacidad visual es previa al ingreso al sistema educativo. Quizás no sea casual que, durante toda su trayectoria escolar, no se hayan interesado por disciplinas científico-matemáticas, manifestando preferencia desde pequeños por asignaturas como 
54 IDENTIFICACIÓN DE NECESIDADES DE APOYO PARA EL APRENDIZAJE Y LA PARTICIPACIÓN DE ESTUDIANTES UNIVERSITARIOS CON DISCAPACIDAD VISUAL: UN ESTUDIO BIOGRÁFICO NARRATIVO - Á. Núñez, M. López

lenguaje y comunicación e historia, y por carreras universitarias del área humanidades y ciencias sociales. También las artes son citadas, como relata Alfonso:

Siempre me gustó historia, lenguaje y música. No siempre tuve música, pero cuando tuve, la aprovechaba al máximo, me ofrecía a ayudarle a los profes. (Alfonso, primera entrevista)

Los participantes dan cuenta de ciertos estereotipos o ideas en torno a lo que pueden hacer las personas con discapacidad visual que se han ido construyendo e instalando, y que de alguna manera han sido parte de sus propias experiencias. En el caso de Gaspar, aparecen este tipo de construcciones en la voz de sus padres:

Mis papás querían que estudiara Derecho o psicología, porque las consideraban rentables y aptas para ciegos, yo no quería estudiar ni Derecho ni psicología. (Gaspar, primera entrevista)

Pero también la familia puede contribuir a desafiar dichos estereotipos:

Entonces está lleno de prejuicios, hay estereotipos que se van cumpliendo: ser músico, ser abogado, ser masoterapeuta, y a mí como que quisieron sacarme de todos esos estereotipos ... lo que más abunda es el ciego que estudia Derecho, porque es una carrera muy de memoria, muy de argumento, muy de palabra, no tienes que ver el delito para saber que es delito ... también le sigue música, ahora recién se está abriendo más el campo de la psicología. (Sabina, primera entrevista)

Por otra parte, también surgen relatos de experiencias de maltrato verbal de parte de compañeros de curso, vividas durante la etapa escolar. Ellos asocian el contenido del maltrato con la condición de la discapacidad visual, ya que las conductas de burla, ridiculización, el uso de apodos, tenían como objeto características físicas propias de dicha condición: el uso de gafas o de parches en los ojos, por ejemplo. Pero también se describen situaciones en las que la discapacidad visual influye en la forma que adoptan las dinámicas de acoso. En el caso de Alfonso, sabía que se burlaban de él, pero desconocía el contenido de las burlas: 
... como a mitad de año ocurrió una cuestión de bullying con un grupito de tres, cuatro compañeros que, claro, ellos se burlaban de medio curso, pero... de lo típico, de lo físico, pero a mi nunca me decían lo que se burlaban de mí, lo dibujaban en la pizarra, era como que yo pasaba, venía del baño, venía de gimnasia y era como risas y risas de ellos cuatro, y el resto se tenía que meter para que no lo hicieran o borrar las cuestiones ... pero era incómodo, en clases, te tiraban cosas, no sé, no era evidente ... eran cosas como por debajo, que a mí me afectaban, pero no tenía después cómo demostrar que lo hacían. (Alfonso, primera entrevista)

Los participantes manifiestan haber vivido estas experiencias con pena, haberse sentido afectados, haber esperado más apoyo de parte de los profesores para controlar las situaciones y detener los comportamientos de acoso de parte de los compañeros. Uno de ellos comparte el hecho de que, en su colegio, donde el acoso aparentemente fue una práctica extendida durante un tiempo, los profesores y autoridades tomaron medidas solo luego de que una estudiante de otro curso se suicidara a raíz de la experiencia repetida de hostigamiento e intimidación. Consideran que ha impactado en la forma en que enfrentan actualmente situaciones similares, aun cuando estas sean de una intensidad menor o no tengan un carácter malintencionado. No obstante, los participantes tienden a matizar y a relativizar la gravedad de las experiencias vividas, al situarlas como una experiencia común y compartida por la mayoría:

Igual, los niños son súper crueles, en general, tampoco fui como "bullyiada", así como que me discriminaran en un grupo de trabajo porque no veía, siempre a todos los niños los molestan por algo, porque es muy flaco, que es muy alto, que es muy blanco, colorín. Entonces como que siento que, claro, yo no tenía ninguna otra particularidad y cuando me molestaban "hola, cinco ojos", como que queda dentro de lo que es un niño chico... Eso en un niño de 10 años es normal. (Sabina, primera entrevista) 
Necesidades de apoyo para el aprendizaje y la participación

Todos los participantes hicieron mención de elementos arquitectónicos fundamentales para su desplazamiento al interior de la universidad. Destaca la necesidad de líneas guía y de rampas bien diseñadas que, más que reemplazar a las escaleras, sean una alternativa al uso de estas. Se relacionó también la idea de accesibilidad con la posibilidad de optar por el uso de uno u otro medio, como manifiesta Sabina:

Accesibilidad quiere decir que yo pueda tomar la decisión de usar las escaleras, que también haya ascensores, si los necesito, que si hay tarimas en las salas entonces también haya la posibilidad de usar una rampa. (Sabina, segunda entrevista)

La señalética, además de facilitar la orientación en el espacio, cumpliría una función de seguridad, que resultaría crítica al permitir fluidez en el desplazamiento. Se resalta la importancia de señalizar cambios de superficie, que las líneas guía conduzcan hasta las salas de clases, que estas últimas lleven en braille sus números o señales de identificación:

... es que hay piletas que están como a ras del suelo, sin una baranda ni nada y eso es súper peligroso, porque si no ves que eso está ahí, te puedes caer. No: estas piletas (que estoy imaginando) son para mirarlas no más, son de paso, entonces tienen baranda. (Sabina, segunda entrevista)

Se aprecia preocupación entre los estudiantes por la amplitud e iluminación de los espacios comunes, tanto de los espacios por los cuales se transita como de aquellos en los cuales se permanece por un periodo más prolongado, en ocasiones con fines de estudio y aprendizaje, como es el caso de las bibliotecas. Por ejemplo, Alfonso manifiesta:

Yo creo que los espacios comunes deben ser amplios, es lo más importante, porque tiene que ir acorde a la cantidad de alumnos, a la necesidad que tienen esos alumnos, no puedes poner una biblioteca que tenga 60 computadores si tienes 1.200 alumnos Y, sobre los baños, pucha que haya varios, que estén ubicados en un lugar no tan difícil, 
porque de repente andas en el $4^{\circ}$ piso y tienes que bajar al $1^{\circ}$. (Alfonso, segunda entrevista)

Es interesante notar que, a este respecto, la preocupación de los participantes no apunta exclusivamente a las personas con discapacidad, sino que manifiestan dificultades generales, fácilmente apreciadas o compartidas por cualquier estudiante de la universidad a la que asisten. Asimismo, plantean como relevante la provisión de guía y entrenamiento para la orientación y la movilidad al interior de los campus universitarios, para poder conocerlos desde el inicio:

No contamos con una persona que nos muestre cómo es la estructura de este lugar. Sí contamos con el caballero que está en la biblioteca, que él nos enseña dónde está el espacio de cada cosa, pero falta el que está en el hall, preocupado de que tú te hagas un mapa mental de cómo es la universidad. (Daniel, segunda entrevista)

Por otra parte, el equipamiento y los espacios para el aprendizaje constituyen una dimensión crítica, tanto para las clases como para el tiempo dedicado al estudio fuera del horario de clases. Esta dimensión contempla también el acceso a los materiales de estudio. La ausencia de rampas dificulta el desplazamiento con bastón y en silla de ruedas, y las superficies irregulares representan un peligro. Asimismo, resulta relevante el acceso al sonido y la adecuada iluminación como factores que favorecen el acceso al conocimiento:

La sala contaría con un micrófono para el profesor y con unos estéreos para que lo que el profesor vaya hablando se escuche en toda la sala, en la parte de adelante, en todas partes, porque hay profesores que hablan muy bajo, entonces esta sala permitiría que uno, sea donde sea que se siente, escuche al profesor. (Daniel, segunda entrevista)

El diseño espacial es también relevante en los espacios para el estudio. Cuando se trata de aprender junto con otros, los participantes conciben una sala cerrada como el espacio idóneo, pues debe permitir el diálogo, pero evitar la interrupción a otros estudiantes. Relevan la necesidad de estudiar junto a otros compañeros, que pueden o no tener discapacidad: 
58 IDENTIFICACIÓN DE NECESIDADES DE APOYO PARA EL APRENDIZAJE Y LA PARTICIPACIÓN DE ESTUDIANTES UNIVERSITARIOS CON DISCAPACIDAD VISUAL: UN ESTUDIO BIOGRÁFICO NARRATIVO - Á. Núñez, M. López

También me gustaría que existiesen salas especiales para personas con discapacidad, pero en las cuales te permitan estudiar con un compañero, que sea o no sea discapacitado, que te permita estudiar con él en voz alta y no estar interrumpiendo a tus pares. (Daniel, segunda entrevista)

Se aprecian también elementos relacionados al tamaño y capacidad de los espacios disponibles para el estudio, y a la coherencia en su organización y distribución. Se concibe un espacio físico cerrado para los estudiantes con discapacidad visual que, además de las condiciones señaladas, cuente con tiflotecnología:

... esa sala tendría que, por ejemplo, tener disponibles computadores con el programa JAWS, que es el programa que nosotros utilizamos, y esa sala tiene los enchufes necesarios para que conectemos nuestros notebooks si es que los queremos llevar, esa sala tiene la capacidad para almacenar a todos los alumnos con discapacidad que están adentro de la universidad ... estaría ubicada dentro de la biblioteca. (Daniel, segunda entrevista)

Los participantes explican que el material de lectura para ellos es funcional en dos formatos: braille y audio. Algunos — como Alfonso- distinguen entre ambos, manifestando preferencias por el audio, dado que el braille ocupa mucho espacio y resulta poco práctico en el espacio universitario:

Los materiales para nosotros tienen que ser digitales, el braille no es tan práctico en la universidad, porque es demasiado, ocupa mucho tiempo y mucho espacio. (Alfonso, segunda entrevista)

Algunos acuden a un centro de grabación, perteneciente a una ONG que brinda ayuda a personas con discapacidad visual: llevan allí su material impreso en papel y solicitan que un voluntario registre la lectura del material en un archivo de audio que luego podrán escuchar. La dificultad de este sistema es que demora bastante tiempo, lo que no es compatible con los ritmos de estudio. En la práctica, el acceso a los textos de la bibliografía es una responsabilidad de los estudiantes; algunos han ido construyendo una base de datos propia, que luego comparten con otros estudiantes. Imaginan una 
universidad que se anticipe y cuente con el material bibliográfico en los formatos requeridos por los diversos estudiantes y que incluya textos impresos con caracteres de tamaño grande. Ello iría de la mano con un funcionamiento institucional particular, concertado en términos administrativos y de gestión, en los que académicos y funcionarios deban cumplir compromisos de trabajo, en línea con reglamentos o mandatos institucionales, de manera que dichos compromisos pasen a ser parte de las funciones y tareas permanentes de los docentes. Gaspar imagina esta realidad en su ejercicio de creación de una universidad ideal:

La universidad les exige hoy día hacer el material en todos los formatos. Significa, por ejemplo, en letra grande ... y no lo hacen los profes ah, hay un centro de accesibilidad. Entonces, como la universidad es un centro inclusivo tú mandas el material y te lo transforman en todos los formatos. Yo sé que los profesores tienen como obligación mandar su material a este centro de accesibilidad y lo hacen accesible. Pero esto se hace antes. Se verifican las matrículas, y entonces cuando yo llegué a mi sala estaba todo listo: yo llegué y me pasaron, me contaron que el sitio web del curso tenía accesibilidad, como ocurre en Australia, y entonces todo el material estaba ahí para mí. (Gaspar, segunda entrevista)

En algunas carreras, el material de estudio incluye diversos soportes y formatos que van más allá de los textos o las lecturas: mapas para el estudio de la geografía, alfabeto fonológico para conocer las letras y sus variaciones, ilustraciones para aprender la anatomía del cerebro, etc. El aprendizaje de los participantes se vio dificultado por no disponer de medios de acceso a estas representaciones, aunque buscaran activamente alternativas, a menudo con la ayuda de compañeros o docentes. Aprender neuroanatomía mediante descripciones de los compañeros o de dibujos con relieves hechos con silicona, o aprender el alfabeto fonológico creando un sistema de descripción de sonidos en conjunto con el profesor, son ejemplos de las estrategias generadas.

Un segundo elemento de la equidad en el acceso al currículo son las estrategias de enseñanza. Los participantes explican que una clase universitaria tradicional, en la que el docente expone 
un contenido apoyándose en una presentación de diapositivas, es inaccesible para un estudiante con discapacidad visual. Algunos de los contenidos necesitan ser representados en figuras en tres dimensiones y los mapas en relieve. Por ejemplo:

... yo tenía ganas de aprender, y no me importaba frustrarme ... cuando veíamos el alfabeto fonético, no entendía nada, entonces fui donde el profesor y le dije "pucha profesor, yo no entiendo, ¿cómo lo podemos hacer? Yo tengo ganas de transcribir igual fonéticamente". Me dice "bueno, lo vamos a hacer sin los símbolos, tú me dices cómo lo transcribirías, el fonema que es, y yo los voy escribiendo". Entonces, yo igual hacía transcripciones fonéticas, no conozco los símbolos, pero yo puedo decirte que una " $n$ " en candado tiene una variación que la hace dental. Entonces, funcionó igual. (Gaspar, segunda entrevista)

Este ejemplo ilustra lo que requieren de sus profesores: un involucramiento activo, un interés por sus necesidades y una disposición abierta y creativa respecto de las metodologías de enseñanza. Sabina, por ejemplo, tuvo un profesor de quien, en la primera clase de la asignatura, recibió material impreso con letra grande, perfecto para que ella pudiese leerlo. Ese mismo profesor realizó ajustes razonables a las evaluaciones, en acuerdo con Sabina, para que ella pudiese abordarlas. Pero Daniel cuenta una historia distinta, que es común en su carrera:

... ni siquiera cuando se ven enfrentados por primera vez a un alumno que es ciego se acercan a él para preguntarle cómo es posible evaluarlo o nada. Y, de alguna manera, es el alumno quien tiene que enseñarle al profesor cómo tiene que realizar la clase para que él entienda mejor, porque en realidad los profesores de la facultad escriben casi todo en la pizarra y después se olvidan de que uno es no vidente, y dicen "tienen que anotar esto porque esto es importante". Y ¿qué significa "esto"? ¿Qué es? ¿De qué está hablando? (Daniel, segunda entrevista)

Ante la posibilidad de imaginar una experiencia ideal de acceso al currículo en el contexto de las clases lectivas, Gaspar sitúa al docente en un contexto institucional que le informa, de manera contingente al trabajo de planificación de su asignatura, sobre las características o particularidades de sus futuros estudiantes: 
... hubo una planificación anterior, hay un vínculo potente entre cómo somos los estudiantes; entonces, por ejemplo, los profesores saben con anticipación qué tipo de estudiantes tienen antes de partir el curso; entonces, por ejemplo, si saben que tienen una persona ciega, adecuan esa clase para las necesidades, pero la persona ciega igual pasa por esa clase, y entiende lo que ocurrió en esa clase. (Gaspar, segunda entrevista)

Un tercer elemento, indispensable para lograr la equidad en el acceso al currículo, son las evaluaciones. Los docentes tienden a utilizar pruebas orales frente a la dificultad de abordar una prueba escrita. Las barreras que presenta este formato son dos: el abuso de la modalidad, utilizándolo en la gran mayoría de las asignaturas, y lo perjudicial que puede ser responder evaluaciones solo en esta modalidad, perdiéndose la posibilidad de desarrollar habilidades que se ejercitan con distintos formatos:

... en el fondo, lo que corta el queque son las distintas modalidades de evaluación, onda, por ejemplo, hay evaluaciones que a ti te van a costar más porque eres menos oral, hay otras que te van a costar menos porque eres más oral, entonces, en el fondo, todos tenemos distintos tipos de inteligencias o de habilidades, y hay evaluaciones que potencian más unas y otras (...) dejas de desarrollar ciertas habilidades si nunca te enfrentas a una prueba escrita, tener que redactar, o a otras evaluaciones más prácticas que seguramente hay en otras carreras. (Gaspar, segunda entrevista)

Proponen que sus evaluaciones tengan el formato oficial, pero con ajustes razonables: una prueba escrita puede ser abordada por un estudiante ciego usando su computador con lector de pantalla y audífonos, y para un estudiante con baja visión sería funcional una prueba impresa con caracteres grandes, de dimensiones específicas a sus necesidades de lectura. Además, manifiestan el deseo de ser evaluados con la misma exigencia y nivel de dificultad que sus compañeros, sin disminuir o modificar los contenidos que contemplan las evaluaciones: 
Me gustaría que, para las personas con discapacidad, la evaluación fuera la misma: mismo formato, quizás incorporar alguna adaptación, pero no en el contenido. Y que te exijan lo mismo. El formato, yo puedo hablar por los alumnos ciegos, porque ha pasado que con una prueba escrita te dicen "usted va a oral", pero te lo dicen el día antes. (Alfonso, segunda entrevista)

Gaspar lo imagina en una universidad ideal:

... las evaluaciones operan en función de ciertos estándares: todos tenemos pruebas orales, todos tenemos pruebas escritas, todos tenemos pruebas para la casa, todos tenemos trabajos en grupo, todos tenemos controles, todos tenemos debates, todos tenemos conversatorios, todos hacemos todo. (Gaspar, segunda entrevista)

\section{Cultura y política en la universidad}

Finalmente, se desarrollan algunas ideas, surgidas del análisis, que permiten dar sentido a dos elementos que emergieron en las entrevistas y que parecían importantes para los participantes, contribuyendo a dar forma a su discurso en algunos casos. La primera de ellas es la accesibilidad como valor para todos.

Durante las entrevistas, los participantes cuestionan el carácter exclusivo de los apoyos que reciben —o quisieran recibir- de la universidad en el ejercicio de sus derechos. Piensan que, así como las bibliotecas están llenas de libros, disponibles para todos, incluso para ellos que no pueden leerlos, entonces, ¿ipor qué aquellos insumos de los que ellos pueden beneficiarse no constituyen también recursos de libre disposición para todos y todas? Citan ejemplos reales o imaginarios como sustento a esta idea; algún compañero con déficit atencional, que estudia mucho mejor escuchando un audio que leyendo un texto, o aquel que podría beneficiarse del audio, porque le lleva más de una hora el trayecto hasta la casa. Sueñan con una universidad donde la totalidad de los recursos para el aprendizaje están universalmente disponibles, donde cada uno estudia usando los formatos que le resultan más cómodos y no los que le corresponden en virtud de poseer o no una discapacidad. Todos los computadores dispuestos para los estudiantes están equipados con un lector de 
pantalla adecuado y todos los estudiantes saben utilizarlo, y pueden hacerlo si ello les resulta de utilidad. Todos, y no solo quienes tienen discapacidad visual, pueden estudiar neuroanatomía con las maquetas del cerebro y geografía con los mapas en relieve, para recordar mejor el curso de un río, o esa forma tan extraña descrita por la línea de una frontera. Sueñan también con la disponibilidad permanente y anticipada de los recursos, en un marco en el que todo lo que existe, existe para dar cumplimiento a un derecho.

El segundo elemento se vincula con la relación entre equidad e institucionalidad. A lo largo de la revisión de resultados, se puede apreciar que los participantes dan cuenta de una suerte de "ideal institucional", de un conjunto de elementos que deberían estar contemplados en las políticas de las universidades para convertirlas en instituciones inclusivas propiamente dichas, vale decir, donde tanto las prácticas como la cultura institucional — mandatadas desde la política - estuviesen permeadas por el respeto y cumplimiento del principio de equidad. De cara a los estudiantes, el compromiso institucional con este principio debe reflejarse necesariamente en tres momentos de la experiencia universitaria: ingreso, permanencia y egreso. Al ser interrogado sobre estrategias específicas para el logro de algo como esto, Gaspar despliega un plan que ha venido esbozando durante ambas entrevistas:

Con capacitaciones, con reuniones, con exigencias también, no sé si visualizas que cuando tiene que ver con el proyecto de desarrollo, no te preguntan: se hace no más, entonces, no te van a preguntar si tú quieres o no hacer tus clases en forma accesible. Lo tienes que hacer. Y para eso te tienen que formar. Y entonces eso también implica encarecer los costos, de ciertas cosas, en términos económicos. (Gaspar, segunda entrevista)

Institucionalizar la inclusión es una operación sistémica, implica reestructurar modos de funcionar, por ejemplo: rediseñar los métodos de circulación de información en función de una sensibilidad respetuosa de la diversidad; formar a docentes y funcionarios para facilitar su trabajo; crear centros de recursos para el aprendizaje allí donde solo hay bibliotecas; hacer modificaciones arquitectónicas que, 
64 IDENTIFICACIÓN DE NECESIDADES DE APOYO PARA EL APRENDIZAJE Y LA PARTICIPACIÓN DE ESTUDIANTES UNIVERSITARIOS CON DISCAPACIDAD VISUAL: UN ESTUDIO BIOGRÁFICO NARRATIVO - Á. Núñez, M. López

en algunos casos, pueden ser de gran envergadura. Pero, sobre todo, y nuevamente en palabras de Gaspar, implica marcar un sello a nivel de personas.

\section{Discusión y conclusiones}

Los resultados del presente estudio dan cuenta de la existencia de numerosas necesidades de apoyo para el aprendizaje y la participación de estudiantes con discapacidad visual en la etapa universitaria. Estas se pueden conceptualizar en dos grandes categorías: orientación y movilidad, y equidad en el acceso al currículo. La participación aparece como un eje fundamental, condicionando y posibilitando el aprendizaje. Los participantes informan de la existencia de distintos tipos de barreras. En primer lugar, en el acceso, ya que, en general, no existen vías de ingreso equitativas a todos los programas de pregrado en las instituciones de ESUP. Es necesario mencionar que, al momento de realizar las entrevistas a los participantes, aún no existía la PSU con adecuaciones, modificación que fue introducida para el proceso de admisión 2020. En este sentido, esta modificación representa un pequeño avance en la materia.

Una vez dentro de la universidad, aparecen numerosas situaciones en que los aspectos arquitectónicos o físicos de los centros educativos dificultan la cooperación para el aprendizaje, tales como la inmovilidad de los pupitres y la imposibilidad de usar las bibliotecas de manera grupal. Los espacios de los que se ha dispuesto para que los estudiantes con discapacidad visual puedan estudiar juntos excluyen a los estudiantes sin discapacidad, lo que ha redundado en menos oportunidades de interacción social. Este hecho constituye una barrera importante pues, fuera de los espacios de estudio, a los participantes a menudo les ha resultado difícil interactuar y establecer vínculos con compañeros sin discapacidad. En esta línea, los hallazgos de Southcott y Opie (2016) muestran que, a menudo, las estrategias de adaptación utilizadas por los estudiantes para acceder a espacios comunes implican perder oportunidades de interacción social. Más allá de las características individuales que puedan influir en esta dificultad de vinculación, no puede omitirse el hecho de que la infraestructura de los campus universitarios podría contribuir 
a superar la situación descrita. Además, de acuerdo con Lourens y Swartz (2016), es indispensable combatir el aislamiento social de los estudiantes con discapacidad y procurar que se vinculen con estudiantes sin discapacidad. La diferencia de sus cuerpos a menudo se traduce en experiencias de vergüenza y rechazo, y es deseable que la institución genere estrategias, tales como campañas, para crear conciencia sobre la discapacidad entre estudiantes sin discapacidad o informar a los estudiantes con discapacidad sobre la existencia de actividades sociales accesibles.

Por otra parte, la noción de "participación" también supone la posibilidad de que la voz de los estudiantes sea escuchada y tenga incidencia en las decisiones que afectan su vida (ONU, 2006). En este sentido, Hutcheon y Wolbring (2012) afirman que los estudiantes con discapacidad en la ESUP deben participar de la discusión sobre iniciativas que les competen, pues son quienes más información poseen sobre sus propias necesidades. Reconocer la inequidad experimentada por personas con discapacidad en el pasado es importante para entender por qué necesitan involucrarse en el desarrollo de futuras políticas. Escuchar sus voces parece ser esencial para garantizar que estén completamente incluidos en los campus universitarios (Mullins \& Preyde, 2013). La existencia de una política institucional informada y adecuadamente difundida en el conjunto de la comunidad educativa puede contribuir al aseguramiento del acceso a los apoyos descritos, así como también impactar en el desarrollo de una cultura inclusiva y en el desarrollo de prácticas inclusivas (Beauchamp-Pryor, 2012).

En este sentido, los resultados de este estudio apoyan la idea de que las instituciones deben cautelar tanto las posibilidades de interacción social, como las condiciones que posibilitan la colaboración para el aprendizaje, sea entre pares como entre estudiantes y profesores. En tercer lugar, las prácticas que favorecen el reconocimiento y la aceptación recíprocos, así como la posibilidad de participar en todos los ámbitos de la vida universitaria, contribuyen al logro de una participación plena (Black-Hawkins \& Florian, 2008; Moriña y Carballo, 2020). 
Por otro lado, los resultados dan cuenta del impacto que ha tenido en la trayectoria de los estudiantes la posibilidad de acceder a las distintas formas de registro y comunicación del conocimiento. Sin duda, es en el acceso a los sistemas externos de representación en el que se concentra la mayor parte de las necesidades de apoyo de los participantes. La falta de disponibilidad de material en formatos adecuados para su conocimiento ha ido en desmedro de sus posibilidades de aprender. Ya durante la etapa escolar, el acceso a formas de representación, tales como mapas e ilustraciones, fórmulas químicas o simplemente texto escrito, resultan determinantes en la experiencia de aprendizaje (Southcott \& Opie, 2016; Pozo, 2006), convirtiéndose en barreras en aquellos casos no infrecuentes en que no hay acceso a materiales adaptados o, mejor aún, construidos bajo criterios de DUA (Díez y Sánchez, 2015; Ruiz et al., 2012). Algo análogo ocurre con las clases universitarias tradicionales, sustentadas en elementos visuales, en las que se torna fundamental que el docente adopte un rol activo en la comunicación y explicación de los contenidos. En la práctica, la mayor parte de las clases carecen de metodologías participativas o que promuevan el aprendizaje conjunto. Se resiente también el abuso del recurso de las pruebas orales para evaluar; la necesidad expresada es ser evaluado utilizando los formatos oficiales para cada caso, pero en modalidades accesibles, ya sea para ceguera o baja visión. En este sentido, lo que los estudiantes demandan estaría en estrecha relación con la noción de "ajustes razonables", esto es, modificaciones y adaptaciones necesarias que no impongan una carga desproporcionada (ONU, 2006). De esta manera, los resultados de este estudio confirman los hallazgos de investigaciones internacionales, en términos de que el profesorado está poco formado para atender al alumnado con discapacidad, lo que redunda en asignaturas que no están adaptadas a sus necesidades (López-Gavira et al., 2016; Lorenzo-Lledó et al., 2020; Moriña \& López-Gavira, 2015; Melero, Moriña y Perera, 2019; Southcott \& Opie, 2016; Perera-Rodríguez et al., 2014; Simón et al., 2016). Tal como han planteado recientemente Sandoval et al. (2020), resulta clave conocer las necesidades de los estudiantes, pues, en la medida en que los docentes las conocen, se sienten más preparados y seguros de diseñar sus contenidos de manera tal que satisfagan las necesidades de todas y todos. 
En este sentido, el acceso al currículo como elemento crítico para la inclusión (Díez y Sánchez, 2015; López-Gavira et al., 2016; Moriña \& López-Gavira, 2015; Perera-Rodríguez et al., 2014; Yuval et al., 2004) puede ser entendido como la diversificación del conocimiento entregado a través de distintos sistemas externos de representación, para que todos los estudiantes puedan acceder a los contenidos. Más aún, los resultados de este estudio sugieren la existencia de un vínculo entre el acceso a los sistemas externos de representación durante la etapa escolar y las preferencias ocupacionales que los estudiantes con discapacidad visual van delineando de cara a la ESUP. Es posible pensar que la falta de acceso a las formas de representación propias de una disciplina actúa como barrera al desarrollo de preferencias académicas u ocupacionales por esa disciplina o por carreras asociadas a ella.

Una dificultad asociada a la satisfacción de las necesidades de apoyo mediante ajustes, es que estos se traducen en prácticas segregadoras que, de una u otra manera, acaban marcando diferencias entre aquellos que los necesitan y los que no. En este sentido, el llamado "dilema de la diferencia" (Minow, 1990), que plantea que "la intervención especial es un remedio para la diferencia pero, a la vez, propicia una perpetuación del estigma asociado a ella" (Florian, 2014, p. 28), adquiere cuerpo en la voz de los participantes de este estudio, cuando aseguran que "lo que no es universal no es inclusivo", en alusión a las prácticas de los programas de apoyo que existen en sus universidades o en otras instituciones de ESUP. Precisamente, dentro de las ventajas que ofrecen las prácticas basadas en los modelos de DUA, está el hecho de que conllevaría una serie de beneficios no solo para los estudiantes con discapacidad, sino para todo el alumnado (Díez y Sánchez, 2015; Ruiz et al., 2012; Moriña y Carballo, 2020). Diseñar todos los contenidos desde este enfoque, permitiría promover la participación y el éxito de todos los estudiantes (Bunbury, 2018; Veitch, Strehlow \& Boyd, 2018), sin la necesidad de hacer ajustes importantes para atender necesidades específicas (Lombardi, Murray $\&$ Dallas, 2013).

Vinculado estrechamente con lo anterior, al indagar las necesidades de apoyo con los estudiantes, ellos demandan la 
aplicación del paradigma del DU en todas las esferas de la experiencia universitaria. Desde su perspectiva, este es el enfoque ideal para el abordaje de sus necesidades para la permanencia y el egreso, lo cual es coherente con los hallazgos de Moriña y Carballo (2020), quienes encontraron que la aplicación de los principios del DU para el aprendizaje en la experiencia universitaria se asocia con resultados positivos para profesores y estudiantes, y concluyen que es una metodología docente útil para dar respuesta a la diversidad en el ámbito universitario.

A partir de los resultados, emerge la relevancia de desarrollar procesos formativos con los académicos, que les permitan diseñar clases universalmente accesibles, además de planificar sus asignaturas teniendo en cuenta las necesidades de sus diversos estudiantes. En el mismo sentido, es necesario sensibilizar y capacitar a los equipos administrativos y de gestión para que puedan anticiparse a los requerimientos y necesidades de los estudiantes con discapacidad, en el marco de procesos de transformación institucional más amplios (Lorenzo-Lledó et al., 2020), considerando también que las posibilidades de los estudiantes de lograr una participación equivalente (a los estudiantes sin discapacidad) está determinada por el contexto institucional (Järkestig, Rowan, Bergbäck \& Blomberg, 2016).

Con todo, las conclusiones de este estudio son tres. En primer lugar, la inclusión en la universidad se juega en políticas institucionales, las que pueden estar determinadas desde el nivel nacional o bien ser creadas por la propia institución de ESUP. Lo relevante es que sean efectivamente aplicadas y no den lugar a lagunas normativas, como ha sido evidenciado en la literatura (Da Silveira $\&$ Dos Santos, 2016; Moriña \& López-Gavira, 2015; Perera-Rodríguez et al., 2015). La importancia de la política radica en su capacidad de sostener medidas en el tiempo, trascendiendo gobiernos, rectorados o decanatos que puedan ser más o menos favorables a trabajar por la inclusión. Así, por ejemplo, insertar la inclusión en el proyecto de desarrollo institucional de una universidad debiese proveer recursos para garantizar el acceso, la permanencia y el egreso exitosos de estudiantes con discapacidad. Asegurar el desarrollo de prácticas y 
de una cultura inclusiva depende necesariamente de la creación y el establecimiento de políticas institucionales claras y concretas.

En línea con lo anterior, es indispensable que la comunidad universitaria, en su conjunto, se involucre en favorecer la formación de una cultura y de relaciones inclusivas. Muchas de las dificultades experimentadas por estudiantes con discapacidad en entornos universitarios provienen de prejuicios o indiferencia por parte de docentes o compañeros. Ser reconocido o acceder a la participación plena al interior de una colectividad depende del marco político, pero sobre todo de las relaciones en las cuales los estudiantes se insertan. Por lo tanto, modificar creencias, ideas y discursos negativos o limitantes en torno a la discapacidad, desde la perspectiva de la equidad, es una tarea que compete no solo a las unidades o programas de apoyo, sino también a profesores, compañeros, funcionarios y a la comunidad en general.

Asimismo, es necesario examinar elementos concretos que favorecen o dificultan el acceso a espacios físicos y al currículo. De ahí que resulte crítico evaluar de qué manera la arquitectura de los centros educativos facilita u obstaculiza el desplazamiento de los estudiantes con discapacidad, y que ello se traduzca en planes responsables de rediseño y en mejoras concretas. Finalmente, es prioritario reconsiderar las metodologías de enseñanza utilizadas por los docentes en su práctica diaria. El aprendizaje se vería favorecido si las comunidades pensaran mejor cómo se construye conocimiento, cómo se representan los contenidos y cómo se generan representaciones compartidas al interior de comunidades de aprendizaje.

Por último, podemos mencionar algunas limitaciones que consideramos tuvo este estudio, junto con algunas proyecciones que se derivan del mismo. En primer lugar, la investigadora que realizó las entrevistas se sorprendió al constatar que tenía más cosas en común con uno de los participantes, que dicho participante con los demás. Esta sorpresa permitió tomar conciencia de que se llegó al terreno con el prejuicio de que las personas con discapacidad visual tienen mucho en común entre sí, que se parecen, que hay un algo que les une en 
términos de identidad. Y este hallazgo permitió cuestionar esa idea de la discapacidad visual como eje de la identidad, dejándolo como un elemento entre muchos otros. En segundo lugar, el número de participantes fue reducido y poco heterogéneo en cuanto a las carreras que éstos cursaban, lo que limitó la amplitud de las necesidades que pudieron manifestar en relación con sus procesos formativos. Esto plantea la inquietud de seguir estudiando los artefactos y modalidades preferentes usadas en distintas áreas disciplinares y profesionales, y cómo éstas pueden plantear diferentes necesidades para la formación y la participación de los estudiantes. En tercer lugar, nuestro foco ha estado puesto en estudiantes con discapacidad visual, pero hemos concluido con la necesidad de pensar el currículo universitario y la accesibilidad en general bajo los principios del DU, con lo cual las categorías específicas de discapacidad deberían dar paso a definiciones más amplias, que favorezcan la participación y aprendizaje de todas y todos. Finalmente, en este estudio no consideramos la participación de docentes, aun cuando sabemos el rol clave que tiene su formación y desarrollo profesional en el avance de las culturas y las prácticas inclusivas en la universidad. Con todo, creemos que debemos proseguir en el avance hacia una universidad más inclusiva, de la mano del fortalecimiento de la investigación y el examen tanto de las necesidades de los estudiantes en diferentes áreas, como de los recursos formativos que pueden ofrecer profesores y formadores mediante currículos más accesibles para todos.

\section{Referencias}

AAIDD (2011). Discapacidad Intelectual. Definición, Clasificación y Sistemas de Apoyo Social. Madrid: Alianza Editorial.

Alba, C., Sánchez, P., Sánchez, J. y Zubillaga, A. (2011). Pautas sobre el Diseño Universal para el Aprendizaje (DUA). CAST (2018). Universal Design for Learning Guidelines version 2.0. Wakefield, MA: Author. Traducción al español versión 2.0. (2013).

Angelino, A., Kipen, E., Librandi, A., Mora, M., y Katz, S. (2013). Modos de abrir el debate. Una experiencia de capacitación sobre discapacidad y accesibilidad en las Universidades Públicas Argentinas. Experiencia llevada a cabo por las Universidades de La Plata, Entre Ríos, Comahue y Lujan en el marco de la Comisión Interuniversitaria: Discapacidad y 
Derechos Humanos. En: L. Perez, A. Fernández y S. Katz, Discapacidad en Latinoamérica. Voces y experiencias universitarias. Buenos Aires, Argentina: Editorial de la Universidad de La Plata.

Beauchamp-Pryor, K. (2012) From absent to active voices: securing disability equality within higher education. International Journal of Inclusive Education, 16(3), 283-295.

Black-Hawkins, K. (2013). The framework for participation: A research tool for exploring the relationship between achievement and inclusion in schools. Derecho y Humanidades, 21, 85-110.

Black-Hawkins, K. \& Florian, L. (2008). Achievement and Inclusion in Schools and Classrooms: Participation and Pedagogy. Paper presented at the British Educational Research Association Conference, Heriot Watt University, Edinburgh, September 2008.

Bolívar, A., Domingo, J. y Fernández, M. (2001) La investigación biográficonarrativa en educación. Guía para indagar en el campo. Granada, España: Grupo Editorial Universitario.

Booth, T. y Ainscow, M. (2000). Indice de inclusión: desarrollando el aprendizaje y la participación en las escuelas. Bristol: Centre for Studies on Inclusive Education (CSIE).

Bunbury, S. (2018). Disability in Higher Education-Do Reasonable Adjustments Contribute to an Inclusive Curriculum? International Journal of Inclusive Education, 1-16.

Buontempo, M. (2000). Inserción laboral de graduados universitarios: un estudio desde las trayectorias laborales. Corrientes, Argentina: Universidad Nacional del Nordeste.

Caballo, C. y Núñez, M. (2013). Personas con discapacidad visual. En: M. Verdugo y R. Schalock, (coords.), Discapacidad e inclusión. Manual para la docencia (pp. 259-283). Salamanca: Amarú.

CAST (2018). Universal design for learning guidelines version 2.2. Recuperado de http://udlguidelines.cast.org

Connelly, F. M. \& Clandinin, D. J. (2004). Canadian teacher education in transition. In: Y. C. Cheng, K. W. Chow \& M. C. Magdalena Mok (eds), Reform of teacher education in the Asia-Pacific in the new millennium: Trends and challenges (pp. 35-43). Dordrecht, The Netherlands: Kluwer Academic Publishers.

Da Silveira, L. \& Dos Santos, L. (2016). Trajetória academica de uma estudante com deficiencia visual no ensino superior. Revista Educacao em Questao, Natal, 54(41), 251-274. 
72 IDENTIFICACIÓN DE NECESIDADES DE APOYO PARA EL APRENDIZAJE Y LA PARTICIPACIÓN DE ESTUDIANTES UNIVERSITARIOS CON DISCAPACIDAD VISUAL: UN ESTUDIO BIOGRÁFICO NARRATIVO - Á. Núñez, M. López

Darr, A. \& Jones, R. (2008). The contribution of universal design to learning and teaching excellence. In: Universal design in higher education: From principles to practice. Cambridge MA: Harvard Education Press.

Díez, E. y Sánchez, S. (2015). Diseño universal para el aprendizaje como metodología docente para atender a la diversidad en la universidad. Aula Abierta, 43, 87-93.

Douglas, G., McCall, S., McLinden, M., Pavey, S., Ware, J. \& Farrell, A. (2009). International review of the literature of evidence of best practice models and outcomes in the education of blind and visually impaired children. National Council for Special Education. University of Birmingham.

Florian, L. (2014) What counts as evidence of inclusive education? European Journal of Special Needs Education, 29(3), 286-294. DOI: $10.1080 / 08856257.2014 .933551$

Fuller, M., Bradley, A. \& Healy, M. (2004). Incorporating disabled students within an inclusive higher education environment. Disability \& Society, 19(5), 455-468.

Gitlow, L. (2001). Occupational therapy faculty attitudes toward the inclusion of students with disabilities in their educational programs. The Occupational Journal of Research, 21, 115-131.

Hopkins, D. (2001). School Improvement for Real. London: Routiedge Falmer.

Hugues, B. y Paterson. K. (2008). El modelo social de discapacidad y la desaparición del cuerpo. Hacia una sociología del impedimento. En L. Barton (coord.), Superar las barreras de la discapacidad (pp. 107-123). Madrid. Editorial Morata.

Hutcheon, E. \& Wolbring, G. (2012) Voices of "disables" post secondary students: examining higher education "disability" policy using an ableism lens. Journal of Diversity in Higher Education, 5(1), 39-49.

Järkestig, U., Rowan, D., Bergbäck, E. \& Blomberg, B. (2016). Disabled students' experiences of higher education in Sweden, the Czech Republic and the United States - a comparative institutional analysis. Disability and Society, 31(3), 339-356.

Langorgen, E. \& Magnus, E. (2018). "We are just ordinary people working hard to reach our goals" Disabled student's participation in Norwegian higher education. Disability and Society, 33, 598-617.

Letelier M. (Ed.). (2019). Educación Superior Inclusiva. Santiago de Chile: Centro Interuniversitario de Desarrollo - CINDA, Grupo Operativo de Universidades Chilenas. Recuperado de de: https://cinda.cl/ publicacion/educacion-superior-inclusiva/ 
Lombardi, A. R., Murray, C. \& Dallas, B. (2013). University Faculty Attitudes toward. Disability and Inclusive Instruction: Comparing Two Institutions. Journal of Postsecondary Education and Disability, 26(3), 221-232.

López-Gavira, R., Moriña, A., Melero-Aguilar, N. \& Perera-Rodríguez, V. (2016). Proposals for the Improvement of University Classrooms: The Perspective of Students with Disabilities. Procedia: Social and Behavioral Sciences, 228, 175-182. DOI: http://dx.doi.org/10.1016/j. sbspro.2016.07.026

Lorenzo-Lledó, A., Lorenzo, E., Lledó, A. \& Pérez-Vásquez, E. (2020). Inclusive methodologies from the teaching perspective for improving performance in university students with disabilities. Journal of Technology and Science Education, 10(1), 127-141.

Lourens, H. \& Swartz, L. (2016). Experiences of visually impaired students in higher education: Bodily perspectives on inclusive education. Disability society, 31(2), 240-251.

Luckasson, R., Borthwick-Duffy, S., Buntinx, W. H. E., Coulter, D. L., Craig, E. M., Reeve, A., Schalock, R. L., Snell, M. E., Spitalnick, D. M., Spreat, S. $\&$ Tasse, M. J. (2002). Mental retardation: Definition, classification, and systems of supports (10th Ed.). Washington DC: American Association on Mental Retardation.

McLinden, M., Douglas, G., Cobb, R., Hewett, R. \& Ravenscroft, J. (2016). 'Access to learning' and 'learning to access': Analysing the distinctive role of specialist teachers of children and young people with vision impairments in facilitating curriculum access through an ecological systems theory. British Journal of Visual Impairment, 34(2), 177-195.

Melero, N., Moriña, A. y Perera, V. (2019). Acciones del profesorado para una práctica inclusiva en la Universidad. Revista Brasileira de Educação, 24.

Mella, S., Díaz, N., Muñoz, S., Orrego, M. y Rivera, C. (2013). Percepción de facilitadores, barreras y necesidades de apoyo de estudiantes con discapacidad en la Universidad de Chile. Revista Latinoamericana de Educación Inclusiva, 8(1), 63-80.

MINEDUC. (2017). Documento de trabajo N². Brechas de género en el Sistema Único de Admisión a la educación superior. Centro de Estudios Mineduc. División de Planificación y Presupuesto. Recuperado de: https:// centroestudios.mineduc.cl/wpcontent/uploads/sites/100/2017/06/ DctoTrabajo2Genero.pdf

Minow, M. (1990). Making all the difference: Inclusion, exclusion, and American law. New York: Cornell University Press. 
74 IDENTIFICACIÓN DE NECESIDADES DE APOYO PARA EL APRENDIZAJE Y LA PARTICIPACIÓN DE ESTUDIANTES UNIVERSITARIOS CON DISCAPACIDAD VISUAL: UN ESTUDIO BIOGRÁFICO NARRATIVO - Á. Núñez, M. López

Moriña, A. y Carballo, R. (2020). Universidad y educación inclusiva: recomendaciones desde la voz de estudiantes españoles con discapacidad. Educação \& Sociedade, 41, 1-16.

Moriña, A. \& López-Gavira, R. (2015). Hidden voices in higher education: Inclusive policies and practices in social science and law classrooms. International Journal of Inclusive Education, 19(84), 365-378.

Moriña, A., López-Gavira, R. \& Molina, V. (2017). What if we could Imagine an Ideal University? Narratives by Students with Disabilities. International Journal of Disability, Development and Education, 64,4, 353367.

Mullins, L. \& Preyde, M. (2013). The lived experience of students with an invisible disability at a Canadian university. Journal. Disability $\&$ Society, 28(2), 147-160.

OECD. (2015). Education at a Glance 2015: OECD Indicators. Paris: OECD Publishing.

OMS. (2013). Salud ocular universal. Un plan de acción mundial para 20142019. Ginebra: OMS. Recuperado de http://www.who.int/blindness/ AP2014_19_Spanish.pdf

ONCE. (2012). Informe sobre la ceguera en España. Recuperado de http://www. seeof.es/archivos/articulos/adjunto_20_1.pdf

ONU. (2006). Convención sobre los Derechos de las personas con discapacidad. Recuperado de https://www.un.org/esa/socdev/enable/documents/ tccconvs.pdf

Parrilla, A. (2002). Acerca del origen y sentido de la educación inclusiva. Revista de Educación, (327), 11-30.

Pegalajar, M. (2013). Tiflotecnología e inclusión educativa: evaluación de sus posibilidades didácticas para el alumnado con discapacidad visual. Revista Electrónica de Investigación y Docencia (REID), 9, 8-22.

Perera-Rodríguez, V., Moriña, A., Cortés, M. y Molina, V. (2015). La universidad como espacio educativo inclusivo. Voces del alumnado con discapacidad. V congreso Iberoamericano de Docencia Universitaria. Enseñanza Superior, Innovación y Calidad en la docencia. Facultad de Psicología y Ciencias de la Educación de la Universidad de Oporto (Portugal), junio de 2012.

Pozo, J. (2006). Teorias cognitivas del aprendizaje. España: Editorial Morata.

Riddle, S. \& Weedon, E. (2014). Disabled students in higher education: Discourses of disability and the negotiation of identity. International Journal of Educational Research, 63, 38-46. 
Rose, D., Harbour, W., Johnston, C., Daley, S. \& Albarbanell, L. (2006). Universal design for learning in postsecondary education: reflections on principles and their aplication. Jornal of Postsecondary Education and Disability, 19(2), 135-151.

Ruiz, R., Solé, Ll., Echeita, G., Sala, I. y Datsira, M. (2012). El principio del "universal design". Concepto y desarrollos en la enseñanza superior. Revista de Educación, 359, 413-430.

Sánchez, S., Díez, E., Verdugo, M. A., Iglesias, A. y Calvo, I. (2011). Atención a la diversidad en las titulaciones adaptadas al RD 1393/2007: Adaptación de una herramienta web de autoevaluación curricular basada en los principios del diseño universal para el aprendizaje. En Jornadas de Innovación Docente en la Universidad de Salamanca (Universidad de Salamanca (España). Vicerrectorado de Docencia, (pp 148-155). Salamanca. Recuperado de http://gredos.usal.es/jspui/ handle/10366/112888

Sánchez-Gómez, V. y López, M. (2020). Comprendiendo el Diseño Universal desde el Paradigma de Apoyos: DUA como un Sistema de Apoyos para el Aprendizaje. Revista Latinoamericana de Educación Inclusiva, 14(1), 143-160.

Sandoval, M., Morgado, B. \& Doménech, A. (2020) University students with disabilities in Spain: faculty beliefs, practices and support in providing reasonable adjustments. Disability \& Society. DOI: 10.1080/09687599.2020.1751078.

Sandoval, M., Simón, C., Márquez, C. y Sánchez, S. (2019). Celebrando la diversidad: un recurso que enriquece la educación superior. Orientaciones para el profesorado universitario. Madrid: UAM Ediciones.

Simón, C., Echeita, G., Sandoval, M., Moreno, A., Márquez, C., Fernández, M.L. y Pérez, E. (2016). De las adaptaciones curriculares al diseño universal para el aprendizaje y la instrucción: un cambio de perspectiva. Paper presentado como borrador de trabajo al Congreso Accesibilidad, ajustes y apoyos, UC3M mayo 2016. Proyecto "Madrid sin barreras".

Southcott, J. \& Opie, J. (2016). Establishing equity and quality: The experience of schooling from the perspective of a student with vision impairment. International Journal of Whole Schooling, 12(2).

Strauss y Corbin (2002). Bases de la investigación cualitativa. Técnicas y procedimientos para desarrollar la teoría fundamentada. Medellín: Editorial Universitaria de Antioquia.

Susinos, T. y Calvo, A. (2006). "Yo no valgo para estudiar...". Un análisis crítico de la narración de las experiencias de exclusión social. Contextos Educativos, 8-9, 87-106. 
76 IDENTIFICACIÓN DE NECESIDADES DE APOYO PARA EL APRENDIZAJE Y LA PARTICIPACIÓN DE ESTUDIANTES UNIVERSITARIOS CON DISCAPACIDAD VISUAL: UN ESTUDIO BIOGRÁFICO NARRATIVO - Á. Núñez, M. López

Taylor, S. y Bogdan, R. (2002). Introducción a los métodos cualitativos de investigación. Barcelona: Paidós.

Thompson, J., Bradley, V., Buntinx, W., Schalock, R., Shogren, K., Snell, M., Wehmeyer, M., Borthwick-Duffy, S., Coulter, D., Craig, E., Gomez, S., Lachapelle, Y., Luckasson, R., Reeve, A., Spreat, S., Tassé, M., Verdugo, M. y Yeager, M. (2010). Conceptualizando los apoyos y las necesidades de apoyo de personas con discapacidad intelectual. Revista española sobre discapacidad intelectual, 41(1), 7-22.

Veitch, S., Strehlow, K. \& Boyd, J. (2018). Supporting University Students with Socially Challenging Behaviours through Professional Development for Teaching Staff. Journal of Academic Language and Learning, 12(1), 156-167.

Verdugo, M. y Schalock, R. (coords.). (2002). Discapacidad e inclusión. Manual para la docencia. Salamanca: Amarú Luckasson.

Vygotski, L. (1978). Mind in society: Development of higher psychological processes. Harvard University Press.

Warren, D. \& Hatton, D. (2002). Cognitive development in visually impaired children. In: I. Rapin and S. Segalowitz (eds.), Elsevier's handbook of neuropsychology (pp. 439-538). New York: Elsevier.

Warren, D. (2000). Visual disorders: The psychosocial perspective-Overview. In: B. Silverstone, M. A. Lang, B. Rosenthal \& E. F. Faye (eds.), The Lighthouse handbook on vision impairment and vision rehabilitation. New York: Oxford University Press.

Yuval, L., Procter, E., Korabik, K. \& Palmer, J. (2004). Evaluation report on the universal instructional design at the University of Guelph. University of Guelph. Recuperado de http://www.coles.uoguelph.ca/TSS/ instructional_design/highlights.aspx

Recibido: 03/09/2018

Aceptado: 14/08/2020 